\title{
A Performance Evaluation on Qos-Supporting Multicast Protocol over Conventional Multicast Protocol
}

\author{
Won-Hyuck Choi, Tae-Seung Lee, and Jung-Sun Kim \\ School of Electronics, Telecommunication and Computer Engineering \\ Hankuk Aviation University, Seoul, Korea \\ rbooo@korea.com, thestaff@hitel.net, \\ jskim@mail.hangkong.ac.kr
}

\begin{abstract}
Recently there have been many requirements for reliability from the multicast communication services that have peer-to-multiple or multiple-tomultiple connecting ability unlike the previous peer-to-peer communications. Multicast protocols include the distance vector multicast routing protocol (DVMRP), multicast extension to open shortest path first (MOSPF), core based tree (CBT) and protocol independent multicast (PIM) structures. These protocols use their own local distance algorithms to utilize characteristics of network packets as routing costs in transferring data. However, multimedia applications require more efficient multicast protocols as well as broadband network connection, real-time transmission. To the requirement, the quality of service sensitive multicast Internet protocol (QosMIC) is urged to be used in Internet. The QosMIC supports several quality of service (Qos) properties: reliability, efficient resource management and flexibility of multicast networks. The QosMIC is in distinction from the existing protocols in that the QosMIC presents data in advance. This paper analyzes the QosMIC and CBT by using objective and practical bandwidths in transferring data packets, and measures their performances on a simulation to test such bandwidths.
\end{abstract}

\section{Introduction}

Recently multimedia workstations, distributed systems and high speed communication systems have had remarkable advances. The combination of these systems encourages the distributed multimedia systems to be developed, and not only workstations but also personal computers can transfer the non-text signals such as voice, image, audio and graphic. Such services can be considered as an aim of data transmission implemented on network and transport layers. The transmission protocols define and implement functions to support the atoms of the services. The up-to-date network communications require the higher layers than transport layer to take over appropriate roles. As a result, users will be ultimately able to enjoy several multimedia applications at a time, each application supplying its own characteristic service.

There are increasing requests on the current multicast communications to have reliability on peer-to-multiple or multiple-to-multiple communication services. In addition to it, broadband network connection, real-time transmission and more efficient multicast protocols are also needed to support multimedia applications. Nevertheless, 
the multicast protocols such as the distance vector multicast routing protocol (DVMRP), multicast extension to open shortest path first (MOSPF), core based tree (CBT), protocol independent multicast (PIM) structures, are lack of communication processing performance, bandwidth and quality of service (Qos), which are fundamental requirements on viable multicasting. It is because such multicast protocols use only their own local distance algorithms as routing costs of packets.

This problem urges to introduce multicast group communication into the service layer newly added to Internet. The service layer provides services necessary to next generations of Internet. The multicasting has fundamental role in the service layer. Multicast must transfer similar information to multiple receivers. This fact results in that single multicast group might suffer from transmission delay which is a defect to unicast communication, since multiple packets are moved through network at a time. Moreover, the quality of user receiving has to be assured.

Network communication must be considered with respect to social exchange, value of the communication and dependency on service. Such properties are sustained by consistent management of Qos layer. The existing multicast routing protocols cannot implement applications to provide such Qos properties. Most of the protocols adopt the PIM, CBT and BGMP as the best efficient structures of Internet. The protocols present users with single path routing. If there is not sufficient resource, they cannot satisfy user's requirements.

Multicasts are divided into conventional methods and methods to improve network service. A representative method of conventional methods is the CBT. The CBT is the most effective method of the existing multicasts to enhance network speed, but it does not consider Qos. Qos enables resource of a source to be connected in shorter time when the source is disconnected from a receiver of network. Therefore, the multicast to present Qos can provide more reliability by preparing sufficient resource.

In this paper, the authors attempt to evaluate a Qos application by using a reliable and efficient multicast protocol, the quality of service sensitive multicast Internet protocol (QosMIC), on Internet. The multicast protocol does meet three requirements: (1) Qos support, (2) reliability, and (3) efficient resource management. The QosMIC is based on multiple routing and Qos supporting, so involves in multicast group connection and fully satisfies the multiple routing and Qos requirements. To compare the QosMIC and the CBT, the best popular one of the existing multicast protocols, a simulation is conducted $[1,5]$.

The hereafter composition of this paper is as follows: section 2 describes the overview of multicast protocols, section 3 describes the QosMIC method, section 4 reports the result of the simulation to compare the QosMIC performance and CBT, and section 5 finally concludes our work.

\section{Multicast Protocol}

Of the previous multicast protocols, four have been commonly used: the source based tree (SBT) multicast protocol constructs trees for each source, the shared tree (ShT) shares trees with multiple sources, the Intra-Domain establishes trees in the same 
domain as to their locations, and the Inter-Domain establishes trees in different domains.

\subsection{Source Based Tree Protocol}

The SBT is also called the source distribution tree (SDT), or the shortest path tree (SPT) in that it is based on the shortest path algorithm. When trees of the SBT are represented, $(S, G)$ is used to denote the source $S$. The SBT which has a good delay characteristic of traffic is efficient in multicast services like the internet broadcasting that has a few senders but many receivers. The distance vector multicast routing protocol (DVMRP), one derivation of the SBT, establishes the shortest path to the receiver to forward datagrams in multicasting mode. And the multicast extension to open shortest path first (MOSPF) protocol is the one that, as you can infer from its name, extends the second version of the open shortest path first (OSPF) (RFC1583). Its name signifies it is independent of unicast routing mechanism, though it has some similar characteristic to unicast protocols. The PIM uses only the resulting tables without associating with algorithms or protocols that put out an unicast routing table. When the density of a tree with a distribution of group members is considered, the PIM is classified into the dense mode (DM) if the density is high and the sparse mode (SM) otherwise [6].

\subsection{Shared Tree Protocol}

The ShT is denoted as $(*, G)$, where * means the whole sources and G the group. The size of the real tree has the value of $\mathrm{O}(|\mathrm{G}|)$ independently of the number of sources since it is the shared tree. The cost in constructing trees is not expensive, but a serious traffic delay might be caused by the increasing number of sources. The ShT is appropriate when multicast services are supplied in the networks that have somewhat narrow bandwidth traffic but many senders. The core based tree (CBT) is one of the ShT and has a core router on the center of the shared tree. In contrast to the PIM-SM protocol that operates as an uni-direction tree and so has constraints on selecting the optimal routing path, the CBT tree operates as bi-direct tree and has more flexible extensibility of networks in comparison with the existing source-based multicast routing protocols.

The best advantage of the CBT protocol is that the change of overhead to manage trees is not very severe when the configuration of member or source is changed. It is due to single tree shared with all members of a multicast group. In addition to it, the CBT can utilize efficiently resource of network including bandwidth of a link because it does not require periodic information to manage groups as the IGMP does and use its bandwidth only for multicast traffic. On the other hand, the disadvantage of the CBT is that it might cause to increase link cost to establish the whole routing path of a tree on the basis of core router and make a bottleneck at core link to condense traffic to the core. That is, high link load needed for overloading the bandwidth of core link makes a bottleneck. When this situation gets more strained, it might result in the deadlock of core link and lead to the stop of multicast service. 
The PIM-SM protocol uses the rendezvous point (RP) that is shared with the whole receiver routers for each multicast group, and it is used as an uni-direction tree [3].

\section{Protocol Description: The QosMIC}

\subsection{Overview}

The main parts of the QosMIC support functions to select a router. In other words, the QosMIC is defined as collection of Qos routing information about multiple paths. When a new node is selected from a multicast tree, Qos requires the exact path by gathering information. To the requirement, the QosMIC is classified into the most upto-date router. The main parts of the router try to search the adjacent routers from tree. Figure 1 shows the node accessing method of the QosMIC. It has to be noted how the core router cannot be used. In the method, the group member of the near node is active and the router selects the Join, and this results in eliminating the necessity of the cores. The QosMIC is superior to the unicast protocol and core-based tree protocols when such advantage is born in mind [1].

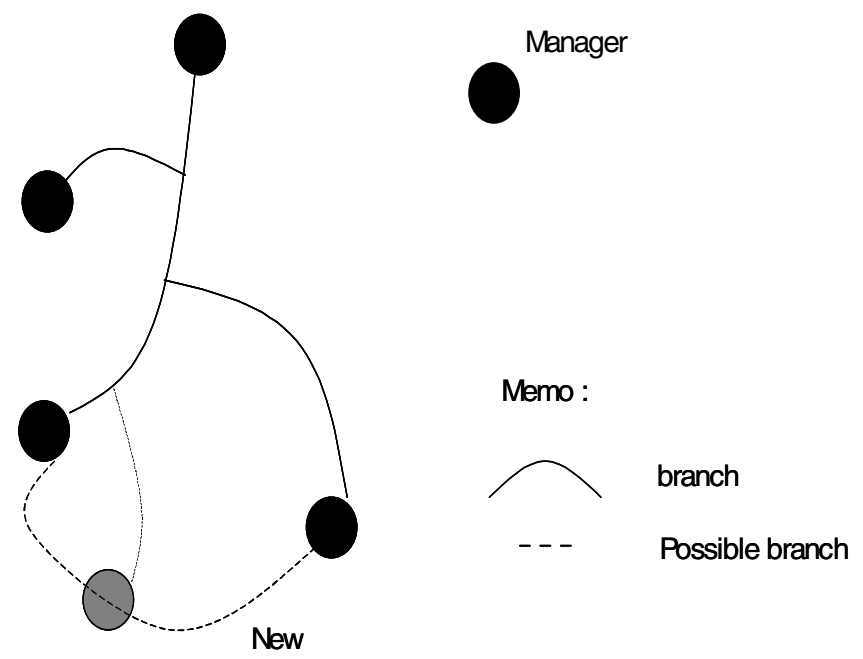

Fig. 1. Approach of the QosMIC 


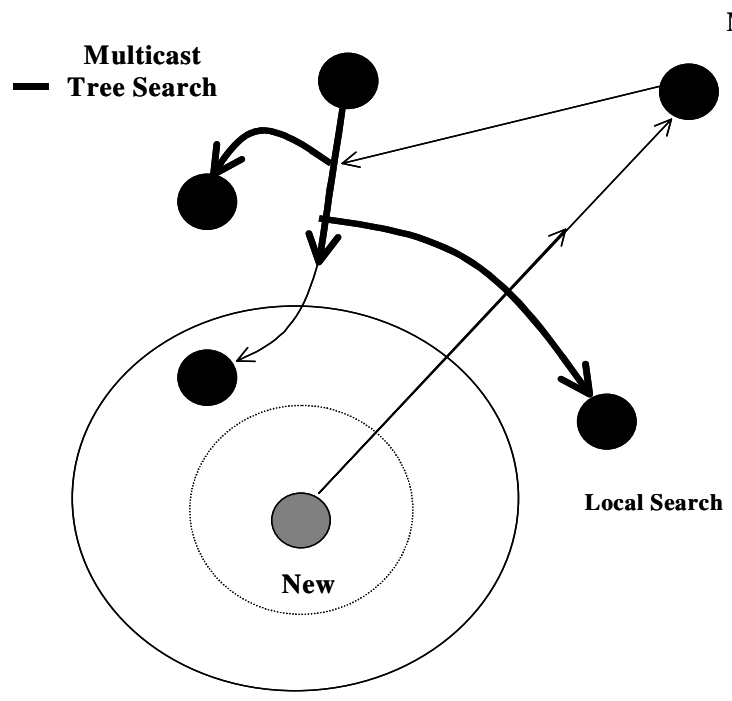

Fig. 2. Searching procedure of the QosMIC

\subsection{Structure and Message Forwarding Method of the QosMIC}

The transmission in the QosMIC structure is implemented as receiver sending on multicast tree and consists of three stages. In the first stage, a router is defined if a tree exists and the tree is able to join other trees through the interfaces of them. These trees are called candidates. In the second stage, the candidates inform the new router of their own information by command messages. In the final stage, the joined router selects nodes that need information. The QosMIC is a source-based tree that Qos characteristic is improved, and is switched such that sharing tree is avoided. The message handling of the QosMIC is implemented as follows. All the routers contain multicast routing information. The information forwards individual multicast packets and executes the appropriate members linked. Each link has entries of tree table in an isolated tree partition. If the packet transferred matches an entry of the routing table, it is linked. In unique tree method, when packets from all sources of a group are matched, the routing entry takes them from shared tree. In $(S, G)$ of the source-based tree, $G$ represents the group and $S$ the source. The shared tree uses $(*, G)$ notation, where $*$ designates the source. The routing location in most multicast protocols of IETF takes lower level. It means that the routing location will be vanished. The adjoining messages correspond with each other periodically and the incoming links from them are newly combined [1], [4]. 


\subsection{Multicast Tree Searching}

When the new router requires a multicast group joined, a new link is added in the multicast tree location and is executed. If the new router already has a group entry, a link is established; otherwise a creator of local searching or multicast tree searching is employed. The creator of router searching is called manager. Manager receives messages from candidate routers of local tree nodes and forwards the messages to multicast routers. This procedure is shown in Figure 2. The candidate selection process is much serious in the QosMIC. The sequential process is conducted like below [8]:

1) Local Search

(1) The New router "floods" a BID-REQ message in its neighborhood. Reverse path multicasting with scope controlled by the time to live (TTL) field. Because QosMIC has the Multicast Tree Search, the TTL value can be kept very small. The advantage should be considered meaningful at the results of simulation conducted later in this paper.

(2) Every In-Tree router that receives a BID-REQ message, becomes a Candidate router, and unicast a BID message back to the New router. The BID message on its way collects information on the expected performance of the path, based on dynamic Qos metrics. The Candidate router considers the New router as a tentative dependent, and cannot leave the tree unless the tentative status is timed out.

(3) The New router collects BID messages. The procedure termincates unsuccessfully, if the New router does not receive any replies before the expiration of a timer set for this purpose. Otherwise, we enter the phase of establishing the connection.

\section{2) Multicast Tree Search}

(1) New router sends an M-JOIN message to the Manager of the group.

(2) The Manager "orders" a bidding session with a BID-ORDER message. Some subset of the routers that receive the BID-ORDER become Candidates.

(3) The Candidates unicast BID's to the New router. The BID's are identical to the BID's in the Local Search.

\section{Simulation}

We compare and analyze the multicast protocols, the QosMIC and CBT described in Sect. 3. The first experiment compares the average speed of traffic flows between terminals when the QosMIC and CBT are used alone. It is to measure the performances in speed, which is the most significant property in networks. The second experiment compares the average delays between terminals. It is to measure how much the Qos between terminals is satisfied when network information is transferred. To do this, a multicast simulation model is established first. The comparison of the QosMIC and CBT routings parameterizes the number and bandwidths of multicast groups, the delay time of packets and the size of data packets as to the characteristic of an appli- 
cation program. The simulation platform is a PC machine with 512MByte memory and $1.5 \mathrm{GHz}$ Intel Pentium Processor, the operating system of the machine the Linux RedHat 7.0 and the tool the Network Simulator - ns Version 2 which is used widely in PC-based environments. In the first experiment, packet sizes to test are 210byte and 512byte.

Figures 3 and 4 report the $4.53 \%$ improvement of the QosMIC with respect to the CBT for 210 byte packets and the $10.25 \%$ for 512 byte packets. The time measurements in the second experiment are the average delay times to the total traffic as with the increasing number of groups between terminals when all nodes linked with a router are considered.

Figures 5 and 6 present the measurements of the average delay times between terminals when the size of packets is 210 byte and 512byte, respectively. It is noted that the delay characteristic of the QosMIC routing is slightly superior to that of the CBT. The delay characteristics of the QosMIC against the CBT are recorded as $12.23 \%$ for 210 byte packets and $14.4 \%$ for 512 byte packets.

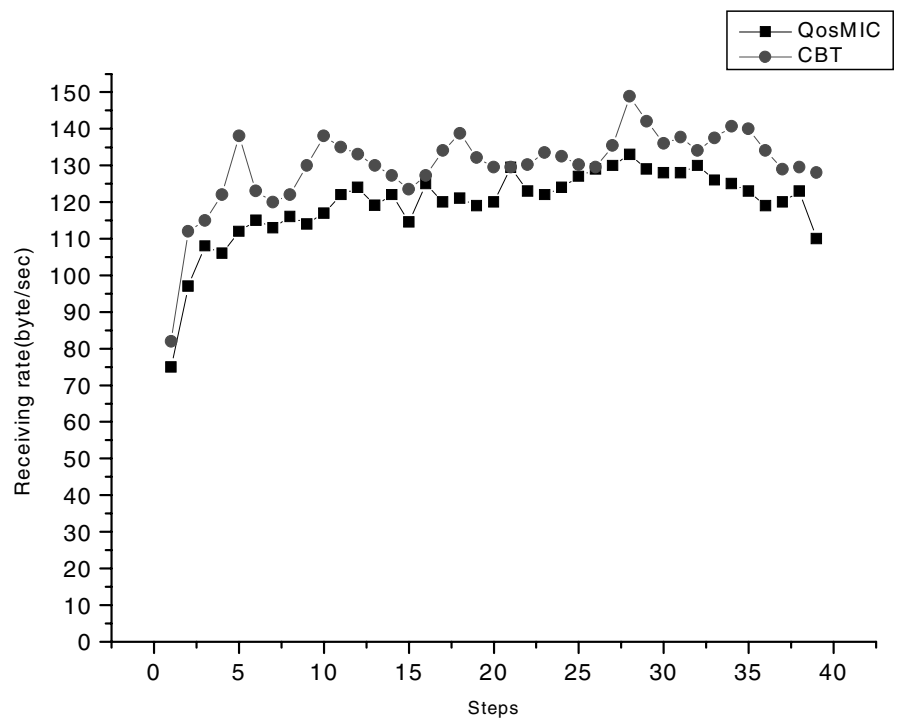

Fig. 3. Data receiving rates for 210 byte packets 


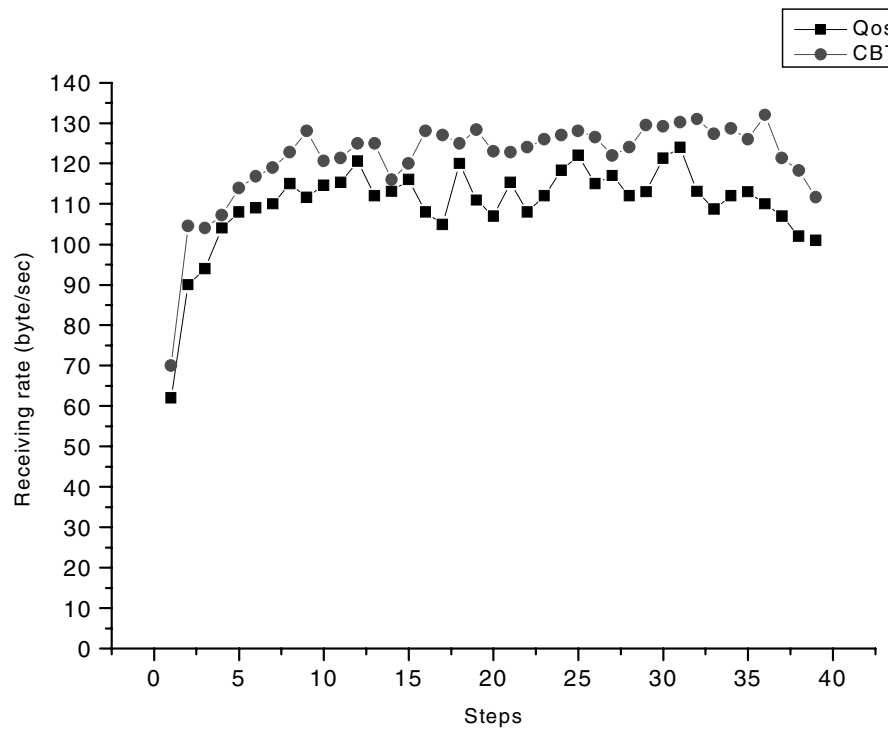

Fig. 4. Data receiving rates for 512 byte packets

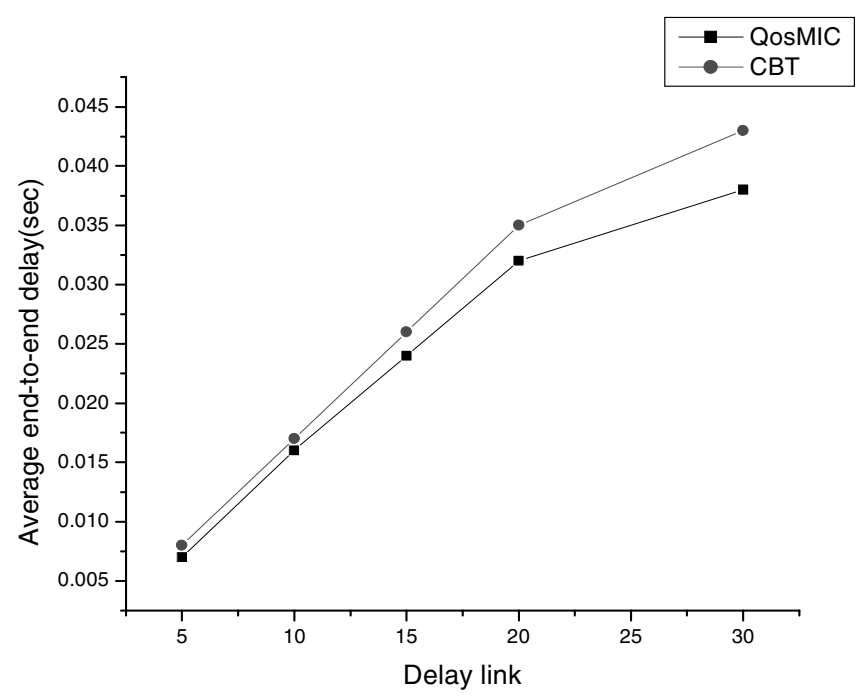

Fig. 5. The average delays between terminals for 210 byte packets 


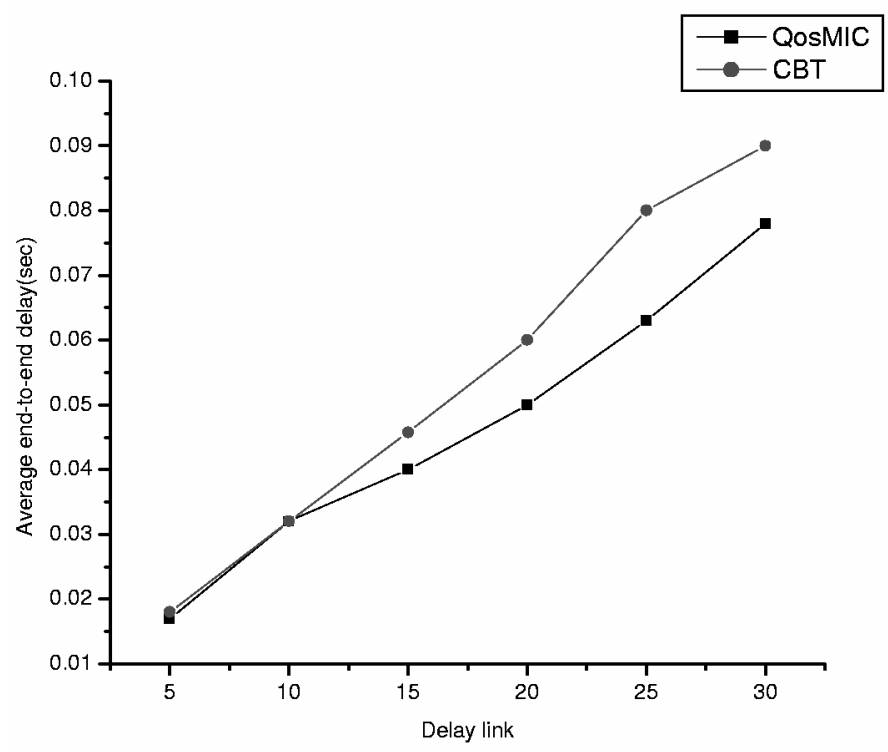

Fig. 6. The average delays between terminals for 512 byte packets

\section{Conclusion}

In this paper, we measured the packet processing times and average delay times between terminals for 210 byte packets and 512 byte packets, respectively. From the first experiment results, the $4.53 \%$ and $10.25 \%$ improvements of the QosMIC against the CBT at the two packet sizes were obtained. And the average delay times were reduced by the $12.23 \%$ and $14.4 \%$. The delay times of the CBT increase more than them of the QosMIC when the number of links of senders is high, the packet size is large and the number of groups of receivers is high. Those evidences strongly confirm the fact that the increasing number of CBT groups induces the increase of the number of groups in Core and the more delays of data packets, and the QosMIC remedies such defect. In the future work, it is required to measure the average delay time and data processing time between terminals as well as the reliabilities on higher levels. Additionally, other multicast protocols beside the CBT must be compared and analyzed in the process between the QosMIC and terminal, and the reliabilities of higher levels be analyzed.

\section{References}

1. The QoSMIC Implementation Web-Site. Available at http: / / www.cs.ucr.edu/michalis/qosmic.html

2. Almerith, K.: A Long-Term Analysis of Growth and Usage Patterns in Multicast Backbone. IEEE INFOCOM. 2 (2000) 824-833 
3. Ballardie, A.: Core Based Trees (CBT version 2) Multicast Routing - Protocol Specification. RFC2189. (1997)

4. Chen, S., Nahrstedt, K., Shavitt, Y.: A QoS-Aware Multicast Routing Protocols. IEEE INFOCOM. 3 (2000) 1594-1603

5. Fei, A., Gerla, M.: Receiver-Initiated Multicasting with Multiple QoS Constraints. IEEE INFOCOM. 1 (2000) 62-70

6. Moy, J.: Multicast Extensions to OSPF. IETF RFC 1584. (1994)

7. Parsa, M., Garcia-Luna-Aceves, J. J.: A Protocol for Scalable Loop-Free Multicast Routing. IEEE J. Select. Areas Comm. 15 (1997) 316-331

8. Yan, S.: Implementation and Evaluation of QoSMIC - A New Internet Multicast Routing Protocol. Master's thesis, Dept. of Elect. Eng., Univ. of Toronto. (1999) 